\section{SEMICONDUCTOR SURFACE AND INTERFACE DYNAMICS FROM TIGHT-BINDING MOLECULAR DYNAMICS SIMULATIONS}

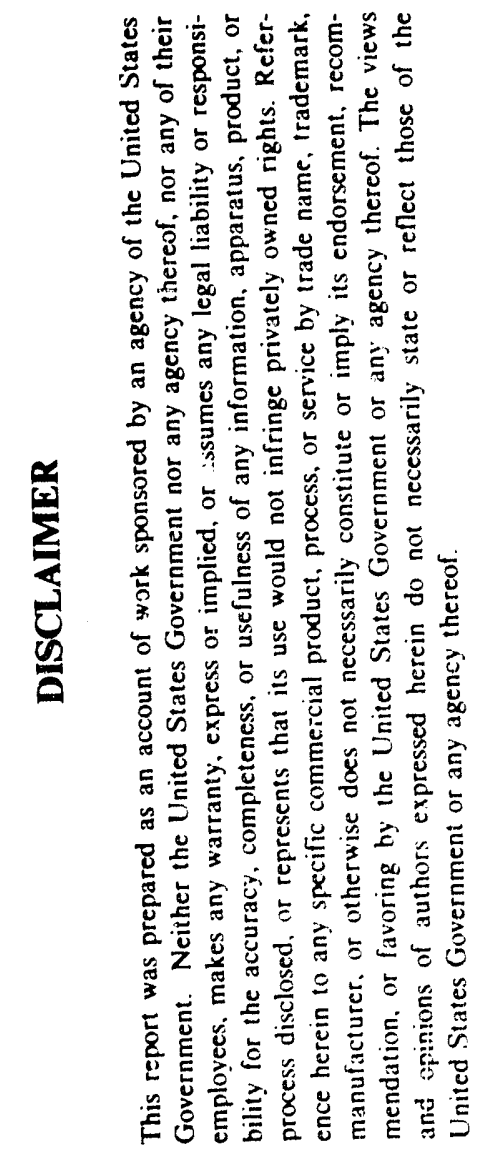

\author{
Presented at the \\ 38th Annual AVS Symposium and \\ Topical Conference \\ Ncvember 11-15, 1991 \\ Seattle, Washington \\ Work supported by \\ the U.S. Department of Energy \\ under Contract DE-ACO6-76RLO 1830 \\ Pacific Northwest Laboratory \\ Richland, Washington 99352
}

G. K. Schenter

November 1991 
Abstract \#: 328

Program \# : SS-TuP48

Put on JVST manuscript

Semiconductor Surface and Interface Dynamics from Tight-Binding Molecular Dynamics Simulations

Gregory K. Schenter and John P. LaFemina

Molecular Science Research Center

Pacific Northwest Laboratory"

P.O. Box 999

Richland, Washington 99352

* Operated for the US Department of Energy by Battelle Memorial Institute under contract DE-AC06-76RLつ 1830 


\begin{abstract}
Tight-binding molecular dynamics simulations have been performed to compute the bulk, (110) surface, and (110)-p(1×1)-S'b(1ML) interfacial atomic vibrational spectra for GaAs and InP. The same tight-binding total energy model which successfully described the static surface and interfacial atomic and electronic structure for these systems is utilized in the molecular dynamics simulations. The results for the bulk vibrational energies are in semi-quantitative agreement with experimental results, displaying approximately the same level of variance as other model computations. Moreover, these simulations are used to examine the effects of anharmonicity in the system by investigating the temperature dependence of the vibrational spectra. The (110) surface vibrational energies are in quantitative agreement with the scattering data, and a comparison of the results for $\mathrm{GaAs}(110)$ and $\operatorname{InP}(110)$ supports the existance of a surface vibrational mode which is characteristic of the relaxed (110) surface, and whose energy is similar for each zincblencie (110) surface. Lastly, the computed vibrational energies for the III-V(110)$\mathrm{p}(1 \times 1)-\mathrm{Sb}(1 \mathrm{ML})$ interface are in semi-quantitative agreement with Raman scattering data and illustrate the effects of the overlayer binding on the surface vibrational spectrum.
\end{abstract}




\section{Introduction}

Quantum-mechanical molecular dynamics (QMMD) simulations, based upon local-density functional1,2 and tight-binding $3,4,5$ (TB) Hamiltonians, have recently been used to examine the structural and harmonic, as well as anharmonic, vibrational properties of Column IV microclusters $2,3,5$ and bulk material4. In addition, molecular dynamics (MD) based simulated annealing computations have been carried out on both clean 6,7 and adsorbed 7 semiconductor surfaces to determine the nature of surface and interface geometries. One advantage to the use of MD simulations over conventional quantum-mechanical computations of total-energy derivative properties is the ability of MD simulations to investigate anharmonicity 4 obtained from a direct dynamical sampling. The applicability of these methods to the study of the vibrational properties of surfaces has been stated in the literature2,3,4 although we are unaware of any published work in this area.

Epitaxical monolayers of Sb on III-V(110) surfaces are of interest in semiconductor surface science because they form ordered, chemically saturated overlayers which are the precursors to metal-semiconductor contacts. Their atomic geometries are known quantitatively from analyses of low-energy electron diffractirn intensities 8,9 and $x$-ray standing-wave studies 10 for $\operatorname{GaAs}(110)$-p(1x1)$\mathrm{Sb}(1 \mathrm{ML}))^{8-10}$ and $\operatorname{InP}(110)-\mathrm{p}(1 \times 1)-\mathrm{Sb}(1 \mathrm{ML}) 10$. Moreover, these geometries have been realicted quantitatively for $\operatorname{GaAs}(110) 11, \operatorname{InP}(110) 11, \operatorname{GaAs}(110)-\mathrm{p}(1 \times 1)-\mathrm{Sb}(1 \mathrm{ML}) 12,13$, and InP(110)-p(1x1)-Sb(1ML)12,13 using tight-binding total energy (TBTE) models which also describe qualitatively the surface electronic states observed by angular- 
dependent photoemission. These studies have shown that the (110) surfaces of zincblende-structure materials exhibit a common relaxation, characterized by a nearly-bond-length-convering rotation of the surface layer of $29^{\circ} \pm 3^{\circ}$ relative to the ideal surface plane ${ }^{14}$. The adsorption of $\mathrm{Sb}$ returns the substrate to a nearly ideal bulk structure through a unique type of chemical bonding between the pi electrons of the top layer $\mathrm{Sb}$ zig-zag chain and the $s p^{3}$ hybrids of the III-V(110) substrate12.

The exploration of the surface atomic dynamics was undertaken initially by Harten and Toennies 15 using inelastic He atom scattering from the $\mathrm{GaAs}(110)$ surface. An "optical" surface mode at approximately $10 \mathrm{meV}$ was soon interpreted by Wang and Duke16 as a characteristic mode of the relaxed (110) surface, corresponding to nearly-bond-length-conserving rotations, about the equillibrium tilt angle, of the top layer relative to the substrate. Doak and Nguyen 17 confirmed and extended the experimental results of Harten and Toennies 15, with emphasis on the extension to surface phonons along the $\bar{\Gamma}-\bar{X}^{\prime}$ line of the surface Brillouin zone. Subsequently, bond-charge slab model calculations of the atomic dynamics of $\mathrm{GaAs}(110)$ and further measurements along the $\bar{\Gamma}-\bar{X}{ }^{\prime}$ and $\bar{\Gamma}-\bar{M}$ lines were reported in an attempt to synthesize the existing data15,17, the rotational surface mode concepts 16 , and the authors' new data into a single, coherent interpretation of the surface dynamics of GaAs(110)18. These calculations suggested that the concept of rigid bond-lengthconserving rotations had limited utility in describing the actual surface normal modes of GaAs(110), despite the fact that the energetics predicted by Wang and Duke were correct. 
The dynamics of the InP(110)-p(1x1)-Sb interface has been recently examined by Hünermann et al.19 via Raman scattering spectroscopy, and localized surface and interface phonons were identified. In a recent study based on a harmonic dynamical matrix treatment of the surface atomic vibrations, Godin et al.20 explored the surface and interfacial atomic dynamics for $\operatorname{GaAs}(110)$ and $\mathrm{GaAs}(110)-\mathrm{p}(1 \times 1)-\tilde{E}$ (11ML) utilizing the same TBTE models used herein, and which were developed previously in static studies of these systems11-13. Using a "restricted dynamical model", in which only the surface layer atoms were allowed to move, Godin et al.20 computed the dynamical force fields and surface atomic vibrational spectra, and predicted relationships between the microscopic force constants, the surface vibrational spectrum and normal modes, and the surface atomic and electronic structure. For the GaAs(110) surface, the computed optical mode energies were in quantitative agreement with the He-scattering results 15,17,18 and the results of the bond-charge slab computation 18 suggesting that the restricted dynamical model provides an adequate description of the surface atomic vibrations. One difficulty asssociated with the computations of Ref. 20 is the need to evaluate numerically the derivative of the total energy for a large number of generalized displacements. As the size of the system to be examined grows, this need can become prohibitive. In addition generalized displacements must be constrained to the harmonic regime. Consequently we have explored the use of molecular dynarnics simulations to compute the desired spectral densities. In our studies, the spectral properties are obtained from dynamical averages instead of direct diagonalization. This provides a 
natural extension of the harmonic dynamical-matrix analysis to the study of anharmonicity in the tight-binding total energy computation of atomic forces.

Our purpose in this paper is to examine the utility of tight-binding based molecular dynamics simulations by computing the spectral density for the bulk, (110) surfaces, and (110)-p(1×1)-Sb(1ML) interfaces of GaAs and InP. The important aspect of our approach is that we utilize the same tight-binding total-energy (TBTE) model to compute the Hellman-Feynman forces that was used successfully to describe the atomic and electronic structure of these systems11-13,21,22. No reoptimization of the model parameters were made in the determination of vibrational properties.

We proceed by describing the TBTE molceular dynamics (MD) model in Section II. The results of the computations are presented and discussed in Section III. We conclude with a synopsis.

\section{Tight-Binding Molecular Dynamics Model}

The equilibrium geometries and forces are computed using the $s p^{3} s^{*}$ tightbinding total energy (TBTE) model developed by Vogl et al.21, Chadi22, and Mailhiot et al.11. In this model the total energy is separated into an electronic "band structure" component and an elastic component of near-neighbor bonds:

$$
E_{T O T}=E_{b s}+U=\sum_{k, n} E_{n}(k)+\sum_{i, j}\left[U_{1} \varepsilon_{i j}+U_{2} \varepsilon_{i j}^{2}\right],
$$


where $\varepsilon_{i j}$ is the fractional displacement from the equilibrium bond distance and $U_{1}$ and $U_{2}$ are determined from bulk structure and moduli. The single particle eigenvalues, $E_{n}(k)$ are taken from an orthogonal nearest neighbor, Slater-Koster23 Hamiltonian. The $\mathrm{k}$ space integration appearing in (1) is accomplished via a quadrature scheme developed by Chadi and Cohen 24 which approximates the integral as a sum of "special k points". All required model parameters are taken directly from previous studies 12,13 . It should be noted that a similar model has been developed by Wang, Chan and $\mathrm{Ho}^{4}$ using a different form of the pair potential, $U$, which represents the ion-ion interactions and the correction for the double counting of the electron-electron interaction. No long -range coulomb forces are contained in the TBTE model. This is not an important limitation of the model for the k-vectors sampled by He-scattering and Raman scattering experiments, nor is it critical to the investigation of trends in phonon dispersion across homologous materials20.

In the computation of the Hellman-Feynman forces to drive the molecular dynamics a single "special point" was used24. The dynamical matrix studies based on the same energy expression have indicated that this approximation results in a $10 \%$ uncertainty in the phonon energies 20 . We find a similar insensitivity of the number of special points to the resulting spectral density as obtained from dynamica! analysis.

In the MD simulation, the Hellman-Feynman forces are calculated with periodic boundry conditions across a non-primative unit cell. Consequently, the frequency distribution obtained from an analysis of velocity autocorrelation of the 
motion corresponds to zero wave vector $\left(q^{\prime}=0\right)$ motion of this non-primative unit cell. In the harmonic limit (low temperature), $3 \mathrm{~N}-1$ modes are expected to be observed where $\mathrm{N}$ is the number of atoms in cell. Due to the symmetry of the crystal, many of these are degenerate. Information of the charictaristic motion in the primative unit cell is contained in the analysis of the non-primative cell, and may be deconvoluted through band folding. In this, the $q \neq 0$ motions of the primative unit cell are transformed to the $\mathrm{q}^{\prime}=0$ motion for the non-primative unit $\mathrm{c} \cdot 11$. For the simulations of bulk GaAs reported in this work the cubic 8-atom non-primative unit cell shown in Figure 1 was used. The use of this non-primative cell allows the motion corresponding to the $\Gamma$ and $X$ phonon modes to be observed in the calculated spectral density. Additional peaks appear which correspond to linear combinations of these modes and are similar to but not identical to the $\mathrm{L}$ motion of the primative cell. For the (110) surface, a 15 atomic-layer slab is used to model the semi-infinite surface (Figure 2). By allowing only the atoms in first two atomic layers to move with the remainder of the layers frozen in their equillibrium configuration, the surface atomic vibrational energies are obtained. One consequence of this approximation is that the surface acoustic modes have non-zero energies at $\mathrm{q}=0$. This is not a serious limitation of the model since we wish to focus on the surface optical modes. Moreover, the use of this restricted dynamical model19 is justified due to the fact that the optical eigenmoues of the surface phonons are dominated by displacements of the surface atomic layer20. In addition, it is at the surface layer where the most significant bonding and eletronic structure changes 
occur.

In the dynamical simulations the Verlet algorithm 25,26 was used to propagate the positions and velocities of atomic nuclei as a function of time within a constant energy ensemble. Initial velocities were distributed according to a Maxwellian velocity distribution corresponding to the simulation temperature. The timesteps for the simulations as indicated in Table I were chosen so that energy was conserved to within $0.05 \%$ of the total energy. Also indicated in Table I are the number of correlation steps and the number of samples for each run, so that the total number of simulation steps is the product of these two numbers. Throughout the course of the dynamical simulation velocity autocorrelation was accumulated as

$C(q, t)=\alpha \sum_{i=1}^{N}\left\langle v_{i}(t) \cdot v_{i}(0)\right\rangle$

where $\alpha$ is a normalization constant, $\mathbf{v}_{\mathrm{i}}(t)$ represents the velocity of the ith atom at time $t$, and the averages were estimated from

$\langle f(i \Delta t) f(0)\rangle \approx \frac{1}{n-i+1} \sum_{j=0}^{n-1} f((i+j) \Delta t) f(j \Delta t)$

Here $\mathrm{n}$ is the total correlation length. A Fourier transform of (2) was made to give an estimate of the configuration averaged spectral density at the end of the simulation. 


\section{Results}

\section{A. Bulk Dynamics}

The phonon frequency distribution at $300 \mathrm{~K}$ and $77 \mathrm{~K}$ for bulk GaAs and at $300 \mathrm{~K}, 77 \mathrm{~K}$, and $2 \mathrm{~K}$ for $\mathrm{InP}$ are shown in Figure 3. As described in the previous section, the use of an 8-atom unit cell in MD simulation results in the frequency distribution containing the phonon frequencies at both $\Gamma$ and $X$. These results are summarized in Table II along with the results of several other computational studies 27,29 and experimentally determined phonon frequencies for comparison 28. As is evident from Table II, the TB-MD frequencies, are in semi- quantitative agreement with the experimental results, with maximum varience for the high energy optical modes. This level of varience is not uncommen however, as is apparent from the results of frozen phonon 29 computations also shown in Table II.

As temperature increases the spectral peaks tend to broaden with the largest spread appearing in the optical modes. Since a completely harmonic system exhibits no spread in spectral density, we attribute the observed behavior to anharmonicity. A slight softening of these modes with increasing temperature is also observed. These effects are more pronounced in the InP spectral densities. A slight hardening of the acoustic modes is observed in botn materials. Again, the differences between the effective harmonic frequencies associated with the larger amplitude high temperature motions and the small amplitude low temperature motions are due to anharmonicities in the system. Care should be taken in interpreting the absolute width of the spectral density due to the intrinsic uncertainties in the model, 
although relative widths and trends across homologous materials are expected to be valid.

\section{B. (110) Surface Dynamics}

The primative surface unit cell of the zincblende-structure (110) surface contains two atoms, each with three degrees of freedom. (See Fig. 2) Consequently, the six $q^{\prime}=0$ modes of the primitive surface unit cell contain two acoustic and four optical modes. As discussed in Section II, the use of a restricted dynamical model causes the acoustic modes to have non-zero energies at $q^{\prime}=0$. For the purposes of this study however, we will focus on the four optical modes.

The computed spectral densities for the GaAs and InP (110) surfaces are shown in Fig. 4, and the surface atomic vibrational energies of the four surface optical modes are summarized in Table III, along with the He-scattering data15,17,18 and tha results of other model computations 18,20 for comparison. It is clear from Table III that the TBMD simulations yield surface vibrational energies that are in quantitative agreement with the available experimental data as well as with the results of other model computations. It is interesting to note that the vibrational energies resulting from a harmonic mode analysis of the GaAs(110)20 surface differ slightly from those obtained from the TBMD analysis, and that this difference is on the order of the uncertainty induced by the use of a single "special" k-point in the evaluation of the band-structure energy. In fact, this difference results from the use 
of different unit cells in the two analyses and thus slightly different integrations performed in the evaluation of the band structure energy and the atomic forces. Given this difference, it follows then that there is no discernable effect of anharmonicity on the energetics of these surface modes. Support for this conclusion comes from the sharpness of the peaks in the computed (110) surface spectral densities (Fig. 4) relative to the peaks in the bulk spectral densities (Fig. 3), for which the anharmonic contributions are significant.

\section{III-V(110)-p(1x1)-Sb Interface Dynamics}

The primitive surface unit cell of the Sb-overlayer also contains two atoms and four optical modes. The Raman scattering studies of Hünermann et al.19 identified four optical modes associated with thr: Sb-overlayer, along with four optical modes they associated with the $\mathrm{Sb} / \operatorname{InP}(110)$ interface. In this work we will examine the optical modes associated with the Sb-overlayer only. Extension of the restricted dynamical model to encompass vibrations in the top two layers will allow for the examination of the interface modes and these computations are currently underway. Preliminary results indicate that there is only a small shift in the computed overlayer-vibrational energies when the top two layers of the slab are allowed to vibrate versus the case when only the overlayer atoms are allowed to 1r.we, providing a posteriori support for the use of the restricted dynamical model.

The spectral densities computed for the IIL-V(110)-p(1x1)-Sb(1ML) interfaces are displcyed in Fig. 5. Table IV contains a summary of these results along with the 
results of the TBTE normal mode analysis for $\mathrm{Sb} / \mathrm{GaAs}(110)^{20}$ and the Raman scattering data for $\mathrm{Sb} / \operatorname{InP}(110)^{19}$. As discussed in Section II, the empirical nature of the TBTE parameterization for Sb overlayers makes these results much less certain than those for the clean surfaces. Nevertheless, the qualitative agreement between the TBMD results and the Raman scattering data demonstrated in Table IV is encouraging. Again, the slight differences in the TBTE normal mode analysis and the TBMD results arises from the slightly different integration schemes used, and indicate that, for these modes, there are no significant anharmonicities in the surface potential energy function.

\section{Synopsis}

Tight-binding molecular dynamics simulations were used to compute the vibrational spectral densities for bulk GaAs and $\operatorname{InP}$, the $\mathrm{GaAs}(110)$ and $\operatorname{InP}(110)$

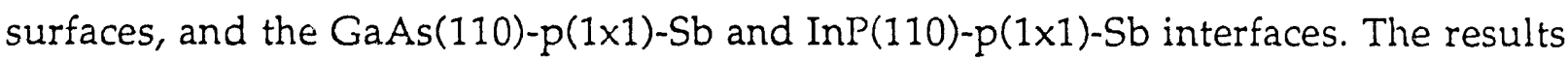
are in quantitative agreement with He-scattering data15,17,18 for the $\operatorname{GaAs}(110)$ surface and in qualitative agreement with scattering data for bulk GaAs and InP and Raman scattering data for the $\operatorname{InP}(110)-\mathrm{p}(1 \times 1)-\mathrm{Sb}(1 \mathrm{ML})$ interface19. Moreover, the dynamical treatment of these systems allows for the investigation of anharmonic contributions to the vibrational energies through the temperature dependence of the vibrational "pectral density. While anhamonic effects were significant in the bulk dynamics, the vibrational energetics of the (110) surface and Sb-interfaces were found to be predominently harmonic. Finally, these studies demonstrate the utility of quartum- 
mechanical molecular dynamics simulations in the investigation of the surface and interfacial atomistic dynamics of semiconductor systems.

Future extensions of this work will focus on the direct determination of the dynamical matrix through generalizations of the velocity autocorrelation function as given by Eqn. 2. In the present study this function corresponds to the trace of the dynamical matrix. Once the dynamical matrix is found, through diagonalization, high temperature generalizations of the eigenmodes are obtained which represent an effective harmonic approximation of the full anharmonic system. 
Table I. Parameters used in simulations

\begin{tabular}{|c|c|c|c|c|c|}
\hline & Material & $\underline{T(K)}$ & $\Delta t(\mathrm{fsec})$ & \# correlations & \# trials \\
\hline \multirow[t]{2}{*}{ Bulk } & GaAs & 300 & 4.0 & 4000 & 90 \\
\hline & & 77 & 4.0 & 4000 & 30 \\
\hline Surface & GaAs (110) & 77 & 4.0 & 4000 & 10 \\
\hline \multirow[t]{2}{*}{ Interface } & GaAs (110)- & 77 & 4.0 & 4000 & 30 \\
\hline & $S b-(p 1 \times 1)-(1 M L)$ & & & & \\
\hline \multirow[t]{3}{*}{ Bulk } & $\operatorname{InP}$ & 300 & 2.0 & 4000 & 20 \\
\hline & & 77 & 2.0 & 4000 & 20 \\
\hline & & 2 & 2.0 & 4000 & 20 \\
\hline Surface & $\operatorname{InP}(110)$ & 77 & 4.0 & 4000 & 10 \\
\hline Interface & $\operatorname{InP}(110)-$ & 77 & 4.0 & 4000 & 20 \\
\hline
\end{tabular}




\section{Table II.}

Comparison of TBMD bulk phonon frequencies with experimental and other theoretical results

\begin{tabular}{|c|c|c|c|c|c|}
\hline \multicolumn{2}{|c|}{ Material } & & \multicolumn{3}{|l|}{$\hbar \omega(\mathrm{meV})$} \\
\hline & & \multirow[t]{2}{*}{ TBMD (300K) } & Experiment (296K) & \multicolumn{2}{|c|}{ Other theory (OK) } \\
\hline & & & Ref. 28 & Ref.27 & Ref. 29 \\
\hline \multirow[t]{5}{*}{$\mathrm{GaAs}$} & $\Gamma$ & $27.4-28.6$ & $33.2-34.8$ & 37.1 & 38.1 \\
\hline & $x$ & 7.0 & 9.8 & & 13.1 \\
\hline & $x$ & $19.7-20.9$ & 27.1 & & \\
\hline & $x$ & $23.0-24.2$ & $28.7-31.4$ & & \\
\hline & "L" & 5.4 & 8.2 & & \\
\hline \multirow[t]{5}{*}{$\operatorname{In} P$} & $\Gamma$ & $29.5-33.5$ & $37.4-43.0$ & & \\
\hline & $x$ & 6.5 & 8.3 & & \\
\hline & $x$ & 16.5 & 23.8 & & \\
\hline & $x$ & $31-32.5$ & $39.7-41.0$ & & \\
\hline & "L" & 3.8 & 7.1 & & \\
\hline
\end{tabular}




\section{Table III.}

Comparison of TBMD (110) surface phonon frequencies with experimental and other theoretical results

\begin{tabular}{|c|c|c|c|c|c|}
\hline \multicolumn{2}{|c|}{ Material } & \multicolumn{4}{|c|}{$\hbar \omega(\mathrm{meV})$} \\
\hline & \multirow[t]{2}{*}{ TBMD (77K) } & \multicolumn{2}{|c|}{ Experiment (296K) } & \multicolumn{2}{|c|}{ Other theory (OK) } \\
\hline & & Ref.15 & Ref. 17 & Ref. 18 & Ref. 20 \\
\hline \multirow[t]{4}{*}{ GaAs } & 9.4 & 10.0 & 10.0 & $8.9-10.3$ & 9.3 \\
\hline & 16.8 & & & & 16.4 \\
\hline & 23.1 & & & & 22.0 \\
\hline & 24.1 & & & & 22.9 \\
\hline \multirow[t]{4}{*}{$\operatorname{InP}$} & 9.5 & & & & \\
\hline & 22.8 & & & & \\
\hline & 26.7 & & & & \\
\hline & 28 & & & & \\
\hline
\end{tabular}




\section{Table IV.}

Comparison of TBMD (110)-p(1x1)-Sb interface phonon frequencies with experimental and other theoretical results

Material

$\operatorname{TBMD}(77 \mathrm{~K})$

9.7

13.6

17.2

18.5

9.1

13.2

16.5

17.8

InP $\hbar \omega(\mathrm{meV})$

Experiment (296K) other theory (0K)

Ref. 19

Ref. 20

10.0

11.8

16.8

18.9

11.9

19.5

20.0

23.0 


\section{Figure Captions}

Figure 1. Cubic 8-atom non-primative unit cell used in simulations

Figure 2. 15 atomic-layer slab used to model the semi-infinite surface

Figure 3a. GaAs Bulk Spectral Density

Figure 3b. InP Bulk Spectral Density

Figure 4a. GaAs (110) Surface Spectral Density, 77K

Figure 4b. InP (110) Surface Spectral Density, 77K

Figure 5a. GaAs (110)-Sb-(p1×1)-(1ML) Spectral Density, 77K

Figure 5b. InP (110)-Sb-(p1x1)-(1ML) Spectral Density, 77K 


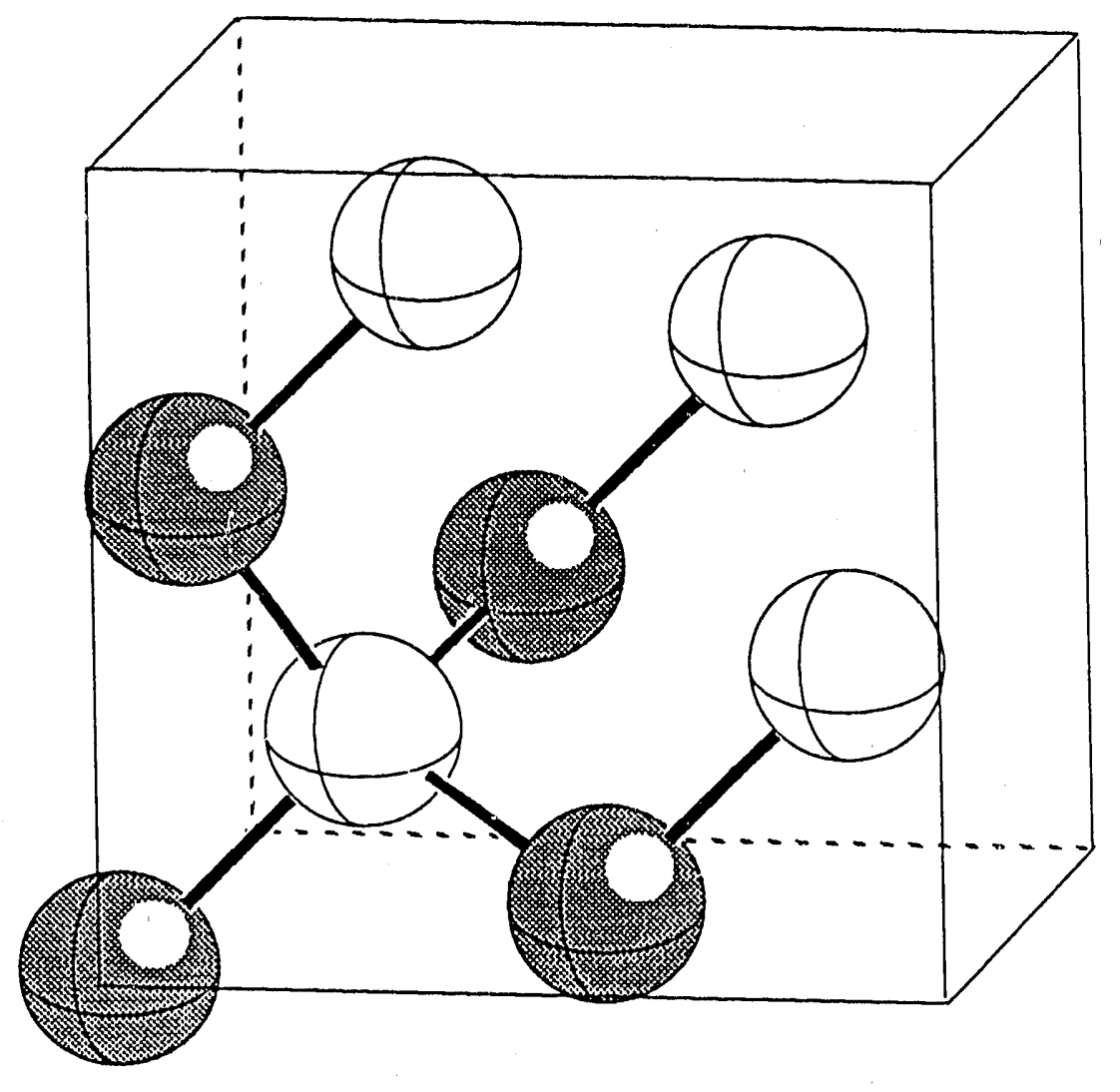




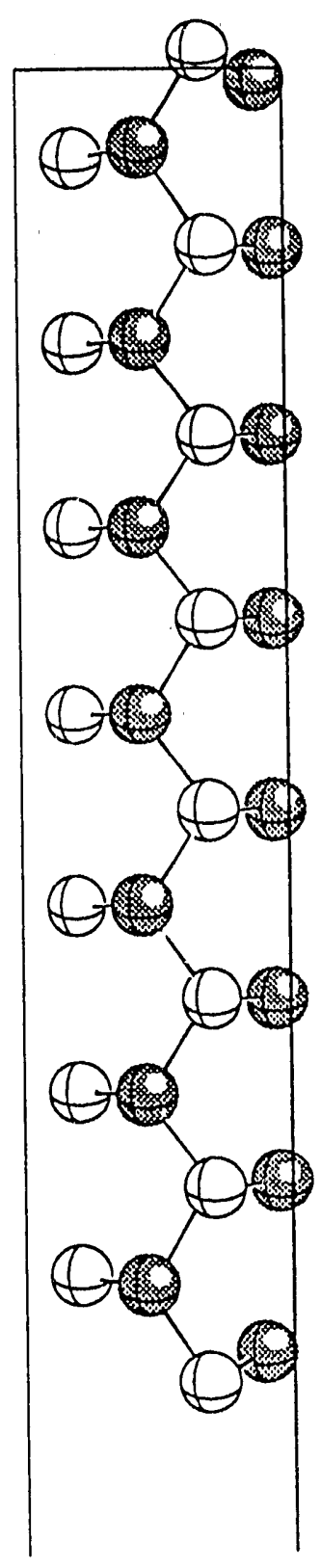




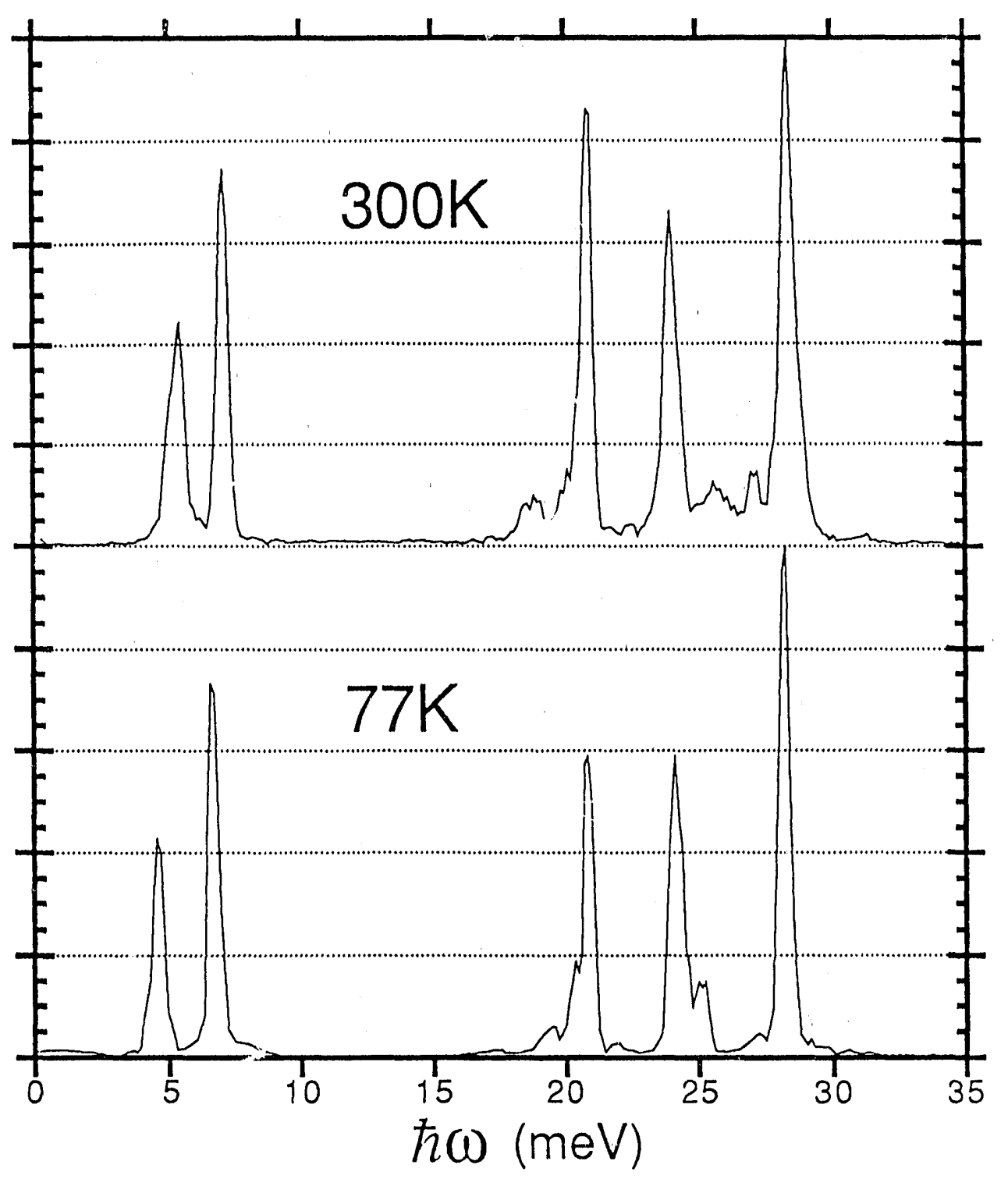




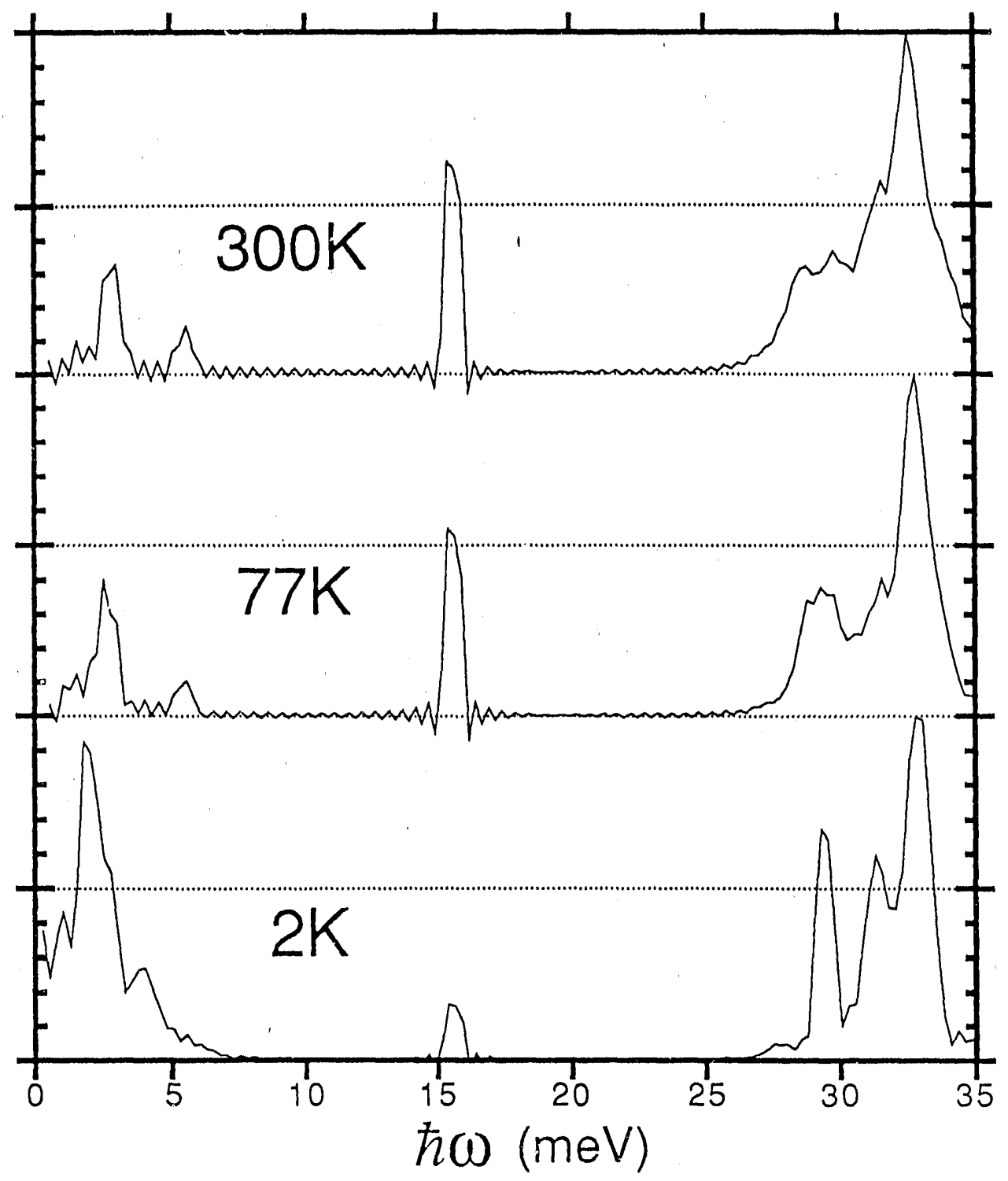




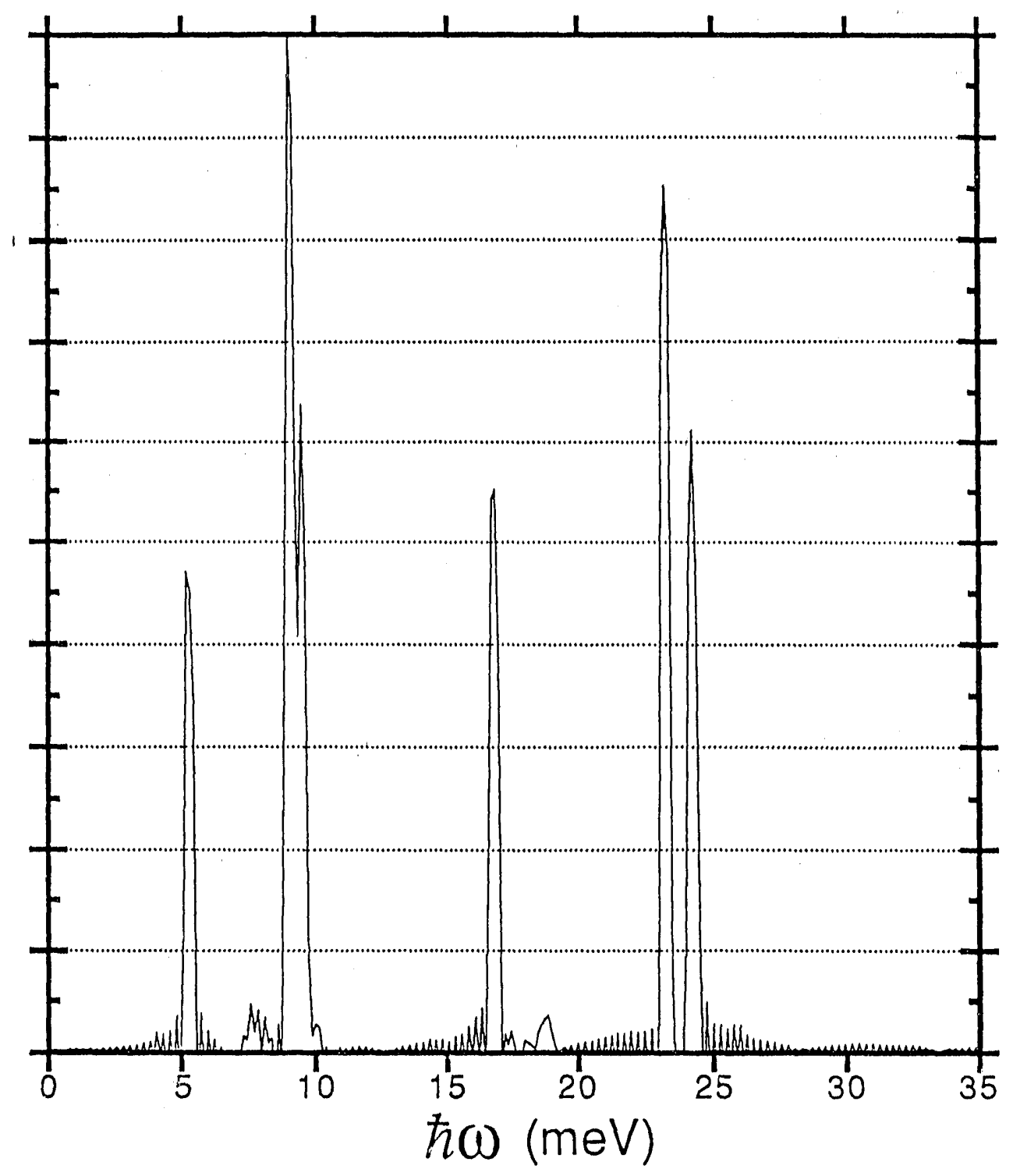




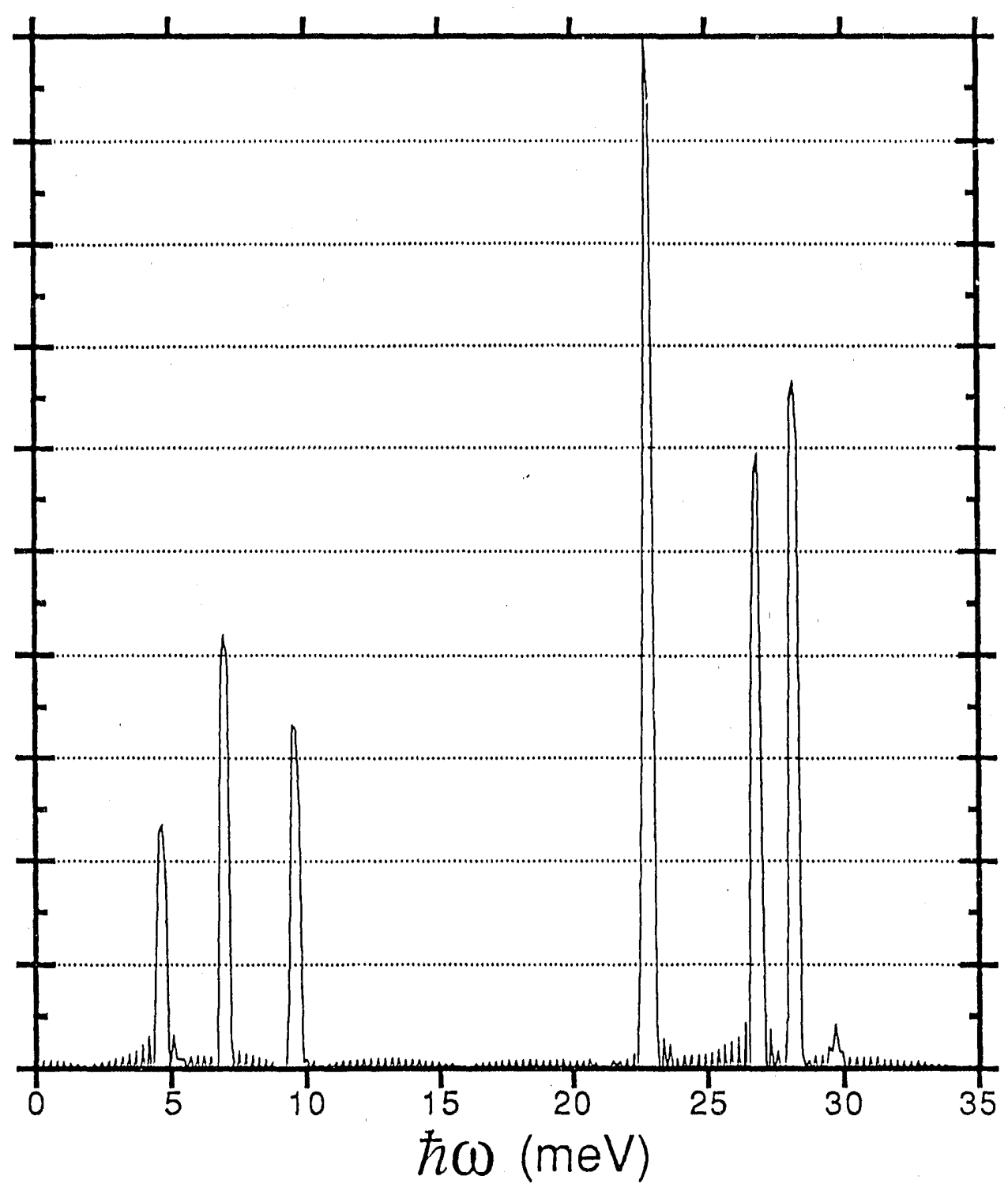




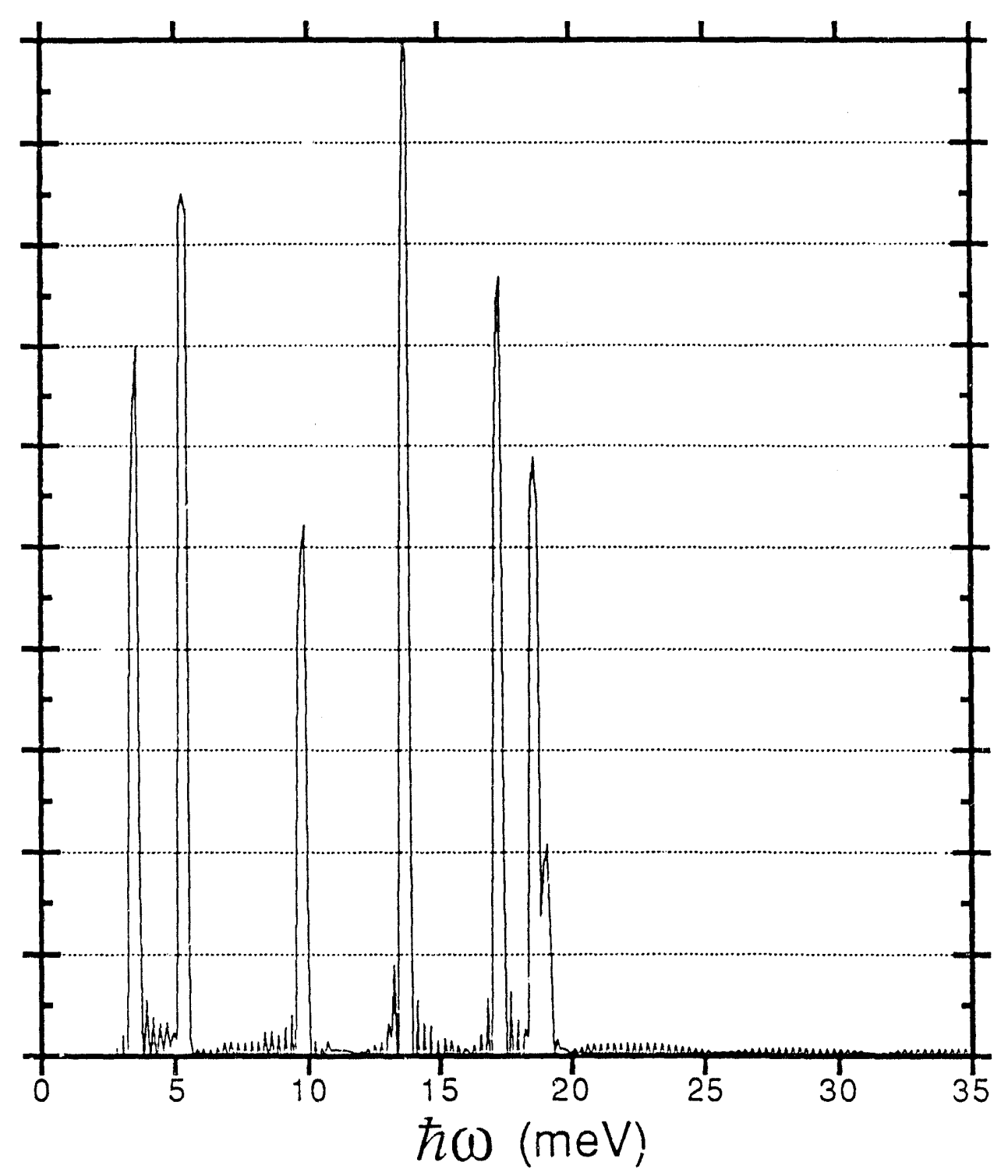




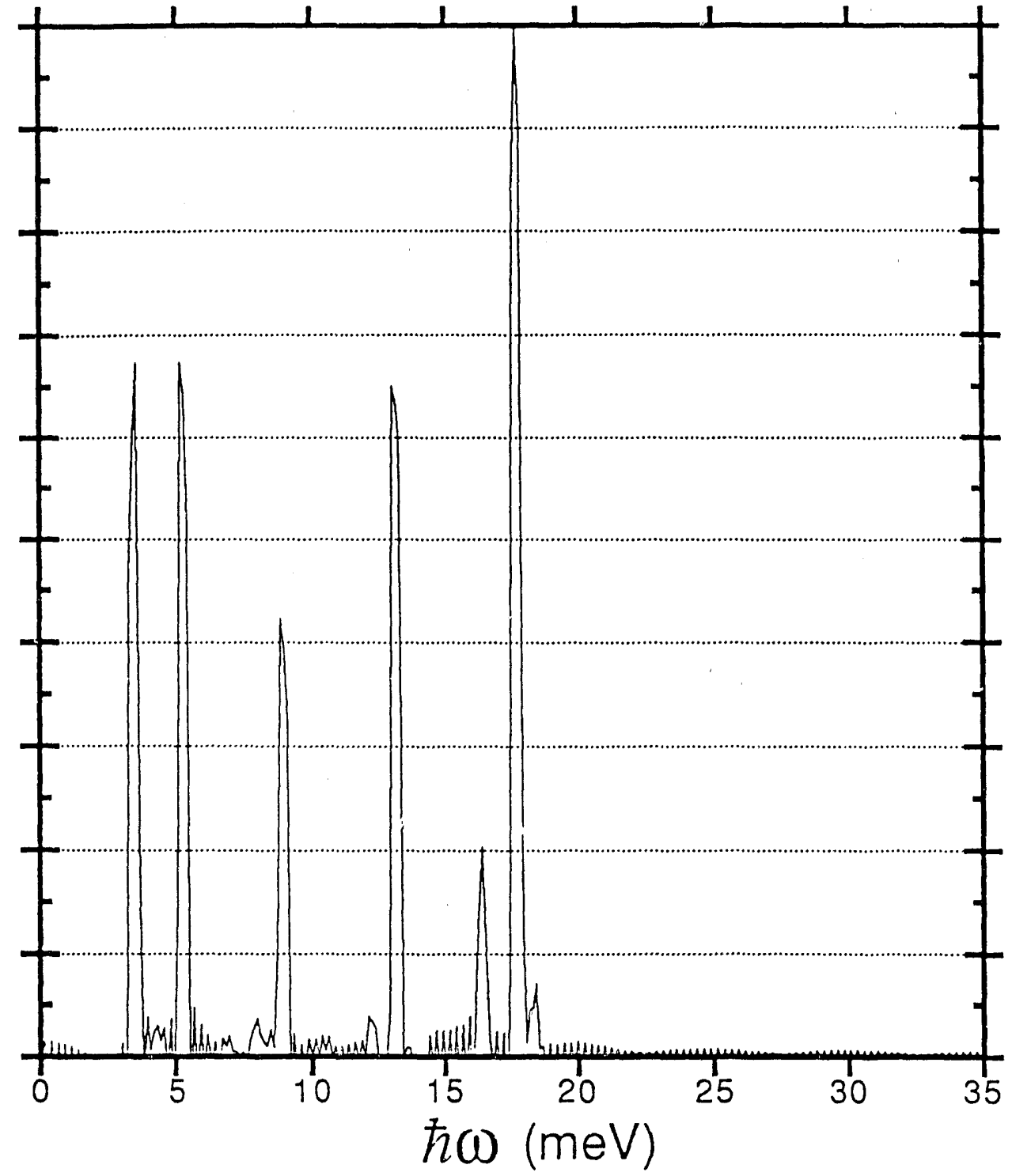




\section{References}

1. R. Car. and M. Parrinello, Phys. Rev. Lett. 55,2471 (1985); 60,204 (1988).

2. (a) O.F. Sankey and D.J. Niklewski, Phys. Rev. B 40,3979 (1989); (b) O.F. Sankey, D.J. Niklewski, D.A. Drabold, and J.D. Dow, Phys. Rev. B 41,12750 (1990); (c) D. A. Drabold, J.D. Dow, S. Klemm, and O.F. Sankey, in Atomic Scale Calculations of Structure in Materials, M.A. Schluter and M.S. Daw, Eds., in press.

3. C.Z. Wang, C.T. Chan, and K.M. Ho, Phys. Rev. B 39,8586 (1989).

4. C.Z. Wang, C.T. Chan, and K.M. Ho, Phys. Rev. B 42,11276 (1990).

5. K. Laasonen and R.M. Nieminen, in Many-Atom Interactions in Solids, Springer Proceedings in Physics 48, R.M. Nieminen, M.J. Puska, and M.J. Manninen Eds., (Springer-Verlag, Berlin, 1990), p264.

6. F.S. Kahn and J.Q. Broughton, Phys. Rev. B 39, 3688 (1989).

7. M. Menon and R.E. Allen, J. Vac. Sci. Tech. B8,900 (1990); ibid B7,729 (1989) and references therein.

8. C.B. Duke, A. Paton, W.K. Ford, A. Kahn, and J. Carelli, Phys. Rev. B 26,803 (1982).

9. W.K. Ford, T. Guo, D.L. Lessor, and C.B. Duke, Phys. Rev. B 42,8952 (1990).

10. T. Kendelewicz, J.C. Woicik, K.E. Miyano, P.L. Cowan, B.A. Karlin, C.E. Bouldin, P. Pianetta, and W.E. Spicer, J. Vac. Sci. Tech. B9,2290 (1991).

11. C. Mailhiot, C.B. Duke, and D.J. Chadi, Phys. Rev. Lett 23,2114 (1984);

J. Vac. Sci. Tech. A 3,915 (1985); Surf. Sci. 149,366 (1985).

12. C. Mailhiot, C.B. Duke, and D.J. Chadi, Phys. Rev. B 31,2213 (1985).

13. J.P. LaFemina, C.B. Duke, and C. Mailhiot, J. Vac. Sci. Tech. B8,888 (1990); in Atomic Scale Calculations of Structure in Materials, M.A. Schluter and M.S. Daw, Eds., in press.

14. C.B Duke, J. Vac. Sci. Tech. A6,1957 (1988).

15. U. Harten and J.P. Toennies, Europhys. Lett. 4,833 (1987).

16. Y.R. Wang and C.B. Duke, Surf. Sci. 205,L755 (198S). 
17.R.B. Doak and D.B. Nguyen, J. Elect. Spec. Rel. Phenom. 44,205 (1987).

18. P. Santini, L. Miglio, G. Benedek, U. Harten, P. Ruggerone, and J.P. Toennies, Phys. Rev. B 42, 11942 (1990).

19. M. Hünermann, J. Geurts, and' W. Richter, Phys. Rev. Lett. 66,640 (1991).

20. T.J. Godin, J.P. LaFemina, and C.B. Duke, J. Vac. Sci. Tech. B9,2282 (1991).

21. P. Vogl, H.P. Hjalmarson, and J.D. Dow, J. Phys. Chem. Solids 44,365 (1983).

22. D.J. Chadi, Phys. Rev. Lett. 41,1062 (1978); Phys. Rev. B 19,2074 (1979); Vacuum 33,613 (1983); Phys. Rev. B 29,785 (1984).

23. J.C. Slater and G.F. Koster, Phys. Rev. 94, 1498 (1954).

24. D.J. Chadi and M. Cohen, Phys. Rev. B 8, 5747 (1973).

25. L. Verlet, Phys. Rev. 159, 98 (1967).

26. M.P. Allen and D.J. Tildesley, Computer Simulation of Liquids, (Clarendon, Oxford, 1989).

27. J.A. Majewski, Acta Physica Polonica A75, 193 (1989).

28. H. Bilz and W. Kress, "Phonon Dispersion Relations in Insulators, Springer Series in Solids State Sciences, Vol 10 (Springer-Verlag, Berlin, 1975) p.105.

29. B.C. Chan and C.K. Ong, J. Phys. Chem. Solids 51,343 (1990). 

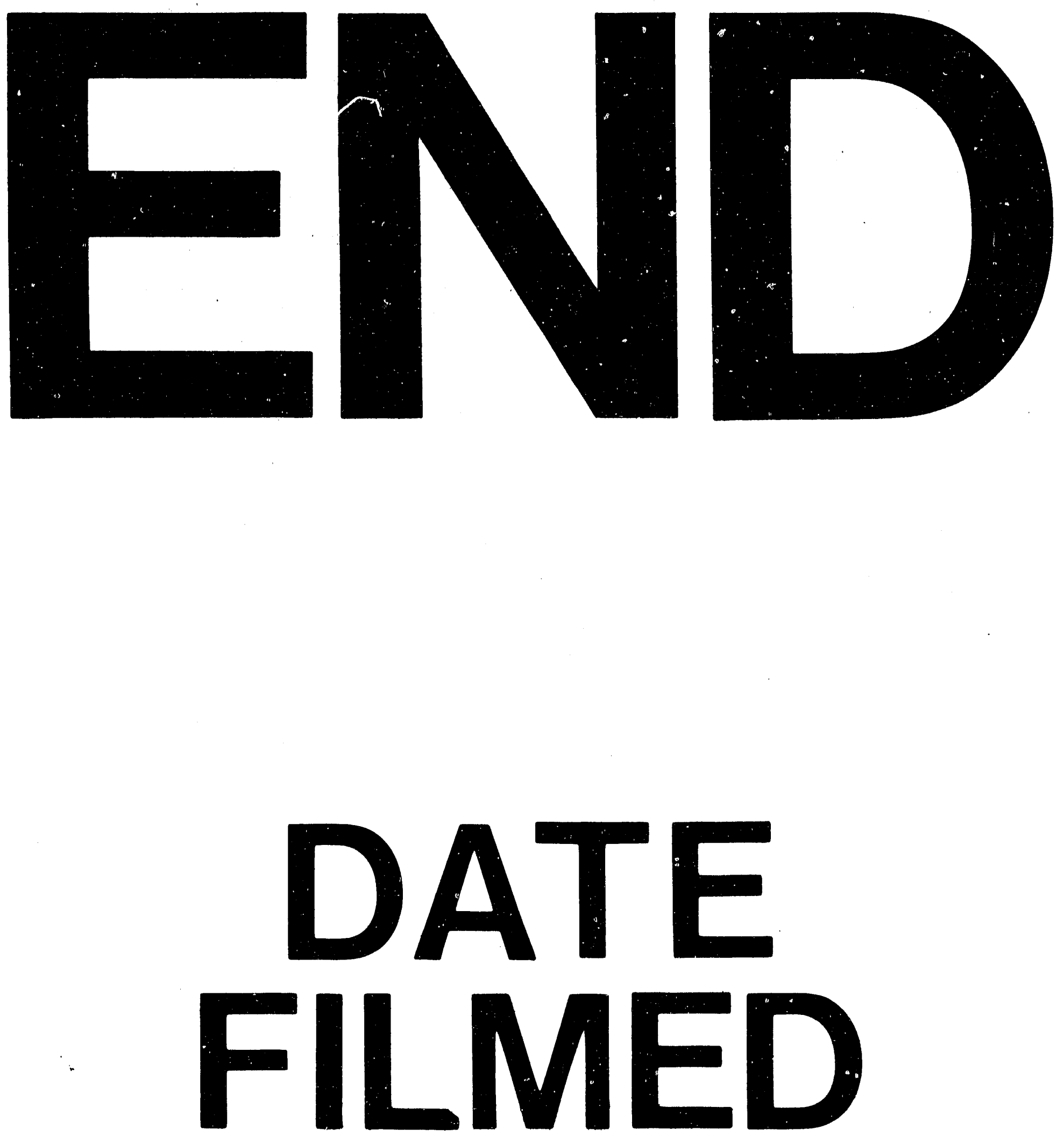

I

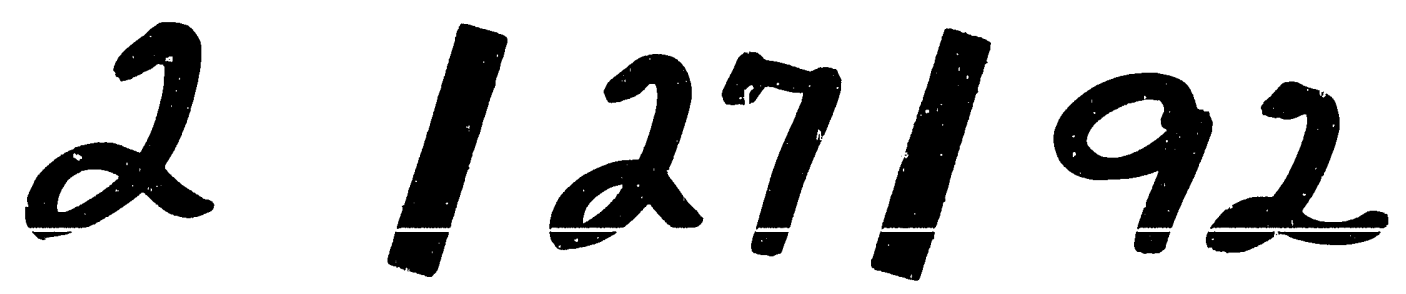


